\title{
A Bird's View on Cell Biology
}

\section{Peter Nick}

Received: 9 July 2010 / Accepted: 9 July 2010

(C) Springer-Verlag 2010

Founded in 1926, Protoplasma is the cell biology journal with the longest tradition. Since over decades cell biology has been focussed on cellular morphology and ultrastructure, the high quality of electron micrographs was always one of its stronger features. Over the last 30 years, cell biology has become more and more functional due to the application of molecular methods. As a consequence, cell biology has merged with other disciplines and therefore, the journal had to face increasing competition by journals from physiology, developmental biology, and biochemistry. Unfortunately, Protoplasma has in the past failed to be attractive enough for authors wishing to have papers published dealing with structure-function relationships at the molecular level. As a result, its impact factor has decreased. It is the expressed wish of the publishers, and the editorial board, to change this situation.

One of the many strategies which can be adopted to achieve this goal is to publish review articles from leading scientists. With this in mind, David Robinson was appointed as Reviews Editor in the summer of 2009. He has been very successful in soliciting reviews; indeed, we are now in the pleasant situation of being able to bring out a special number of Protoplasma devoted entirely to review articles. Depending upon the availability of review articles, we will create from time to time further special numbers of this type.

The review articles in this special number are not thematically related to one another, but illustrate different facets of cell biology all over the tree of life. This broad perspective precludes an editorial comment on their contents. We have reviews on problems related to insects (Clark et al.), red blood cells (Baines), plants (Lütz, Mittag), virus entry (Lin and Guttman, Thorley et al.), as well as a methods-based article on correlative microscopy (Verkade). As a result, we hope that this special number will enjoy a wide readership.

P. Nick $(\square)$

Molecular Cell Biology, University of Karlsruhe,

Institute of Botany,

Kaiserstr. 2,

76131 Karlsruhe, Germany

e-mail: peter.nick@bio.uni-karlsruhe.de 